\title{
Differences in Human Motoneuron Excitability Between Functionally Diverse Muscles
}

Christopher A. Taylor ${ }^{1}$, Tyler E. Kmiec ${ }^{2}$, Christopher K. Thompson ${ }^{2}$

${ }^{1}$ Department of Kinesiology, Temple University College of Public Health

${ }^{2}$ Department of Health and Rehabilitation Sciences, Temple University College of Public Health

Correspondence: taylor.chris@,temple.edu (Christopher A. Taylor)

\begin{abstract}
Introduction: Spinal motoneurons (MN) transmit neural commands from the brain to the muscles they innervate and, as a result, produce functional movement. However, $M N$ s are not simply passive conduits of these commands and, instead, actively shape motor output through alterations in intrinsic excitability. We hypothesize that the excitability of $M N s$ is not fixed across the body; instead, MNs are functionally tuned to the tasks they control. Such findings could indicate a divergent control strategy that allows for the differential regulation of motor pools for specific tasks. We aim to investigate the mapping of MN excitability across motor pools. Methods: High-density surface electromyography of the tibialis anterior (TA) and first dorsal interosseous (FDI) was recorded from four neurologically intact participants while they performed low-level, isometric contractions. The data were decomposed into underlying motor unit action potentials and paired motor unit analyses were subsequently performed on these spike trains to quantify MN excitability ( $\Delta F)$. Results: 1,638 motor unit spike trains were extracted across all contractions. Mann-Whitney $U$ test revealed that all subjects (4/4) had significantly higher maximal discharge rates in FDI, 19.13 [17.62 - 20.59] pps, when compared to the TA, 13.08 [11.51 - 15.46] pps. All subjects (4/4) had a bigher $\Delta F$ in the TA $(4.22$ [2.89 - 5.61] pps) than the FDI (3.62 [1.23 - 5.94] pps), with $3 / 4$ reaching statistical significance. Conclusions: Our findings suggest that the discharge rate and intrinsic excitability of human MNs differs across TA and FDI motor pools during similar isometric tasks. These results support the notion that motor pools are functionally tuned to their environmental demands.
\end{abstract}

\section{Introduction}

Spinal motoneurons $(\mathrm{MN})$ are the conduits for volitional movement in humans, communicating motor commands from the brain through the spinal cord to muscle fibers. ${ }^{1}$ The motor system is organized such that a single $\mathrm{MN}$ and the muscle fibers it controls (i.e., motor unit) represents the smallest functional unit of the motor system. Two accepted mechanisms explain the way by which the human nervous system controls force output: 1) by recruiting more motor units to contribute to the task; 2) by increasing the discharge rate of those motor units already active. ${ }^{2-3}$ These mechanisms have been well-explored and suggest a highly linear, rigidly organized, model for the regulation of motor unit activity. ${ }^{4-5}$ However, more recent investigations show MNs do not simply act as a passive follower of the brain, but instead, have complex intrinsic properties that may cause non-linear changes to motor unit behavior. ${ }^{6-8}$ This active role of the $\mathrm{MN}$ in the generation of muscle force is largely unaccounted for in current models of motor control and understanding of pathologies of the neuromotor system. 
These intrinsic properties of $\mathrm{MNs}$ are regulated via monoamines (i.e., norepinephrine and serotonin) released from the brainstem. ${ }^{9}$ The released monoamines result in intrinsic changes to the excitability of MNs through the activation of persistent inward $\mathrm{Ca} 2+$ and $\mathrm{Na}+$ currents (PICs) on the soma and proximal dendrites. ${ }^{10}$ The functional consequence of this is to allow the $\mathrm{MN}$ to remain active even after descending synaptic drive has lessened or ceased. ${ }^{711}$ A classic example of this is a current clamp recording from a spinal motoneuron from an animal, which is provided a triangular injection of current and hysteresis is observed in the discharge of the neuron. ${ }^{12}$ That is to say, more current is needed to initiate discharge than is needed to maintain repetitive discharge - this extra current and resulting hysteresis is provided in large part by the activation of PICs. It has been proposed that these channels are more prominent in postural muscles, to support the tonic activation of muscles. ${ }^{13}$ Further, there is evidence that disruptions in the regulation of monoamines and PICs may lead to clinically significant symptomology in a wide range of disease conditions including spinal cord injury, ${ }^{14}$ amyotrophic lateral sclerosis (ALS), ${ }^{15}$ stroke, ${ }^{16}$ and sepsis. ${ }^{17}$

Direct observation of transmembrane currents of human MNs is not yet possible. But because the neuromuscular junction is a high-fidelity synapse where each MN action potential results in an action potential of the associated muscle fibers, it is possible to infer underlying cellular process from the discharge of pairs of individual motor units. Further, utilization of noninvasive, high-density surface electromyography (hdEMG) arrays and decomposition techniques ${ }^{18}$ allows for the quantification of the concurrent discharge of a large amount (in some cases $>40$ ) of individual human motor units. Such population measures of human MN discharge provide us with a new means to quantify the distribution of human spinal MN excitability across motor pools. ${ }^{19}$

Here, we map the excitability of human spinal MNs within and across motor pools in the upper and lower extremity. We hypothesize that the excitability of MNs is not fixed across the body, but rather is functionally tuned to the tasks they control. We hypothesis that a $\mathrm{MN}$ that innervates a key postural muscle of the lower extremity (TA) will have greater intrinsic excitability compared to a $\mathrm{MN}$ that innervates an intrinsic muscle in the hand (FDI). Understanding the diverse repertoire of mechanisms available for the central nervous system to control motor output will provide insight for future therapeutic interventions for individuals with neuromotor pathology.

\section{Methods}

\section{Participants}

Four human subjects ( 2 females, mean age $26.5 \pm 4.98$ ) with no known neuromuscular impairments were recruited for this study. All subjects self-reported right hand and foot dominance and provided written informed consent prior to participation. All procedures were approved by the Institutional Review Board at Temple University (Protocol \# 23971). 


\section{Experimental Design}

To investigate the behavior of MNs within and across motor pools of the upper and lower extremity, two experimental sessions were implemented on non-consecutive days. Each experiment consisted of data collection from a single muscle. Similar protocols were conducted during both experimental sessions, with the muscle being tested first randomly assigned. To quantify the discharge characteristics and estimated excitability of individual MNs, a series of slow ramping isometric muscular contractions were utilized, in which the force output could be slowly modulated over time in a consistent manner. During this time, MN activity was recorded with hdEMG.

\section{Experimental Set $U p-L e g$}

Prior to the experimental session, the skin over the dominant-side (i.e., right) tibialis anterior (TA) was shaved, abraded with high-grit sandpaper, and cleansed. One 64-channel high-density electrode array ( $8 \mathrm{~mm}$ inter-electrode distance; OT Bioelettronica; Turin, Italy) was placed on the skin over the muscle, covering the largest portion of the muscle belly, and secured with medical tape (3M Transpore; St. Paul, MN). Participants were seated in an isokinetic dynamometer (Biodex; Shirley, NY) and secured with straps across the torso and upper leg in a comfortable position of $60-90^{\circ}$ hip flexion and $15^{\circ}$ knee flexion. The ankle was secured to a footplate achieving $15^{\circ}$ degrees of plantarflexion and affixed to a six degrees of freedom load cell (JR3; Woodland, CA). The ankle's axis of rotation was centered to the load cell in this position (See Figure 1). Real-time feedback of torque output was provided on a TV monitor positioned directly in front of the participant to assist task performance.
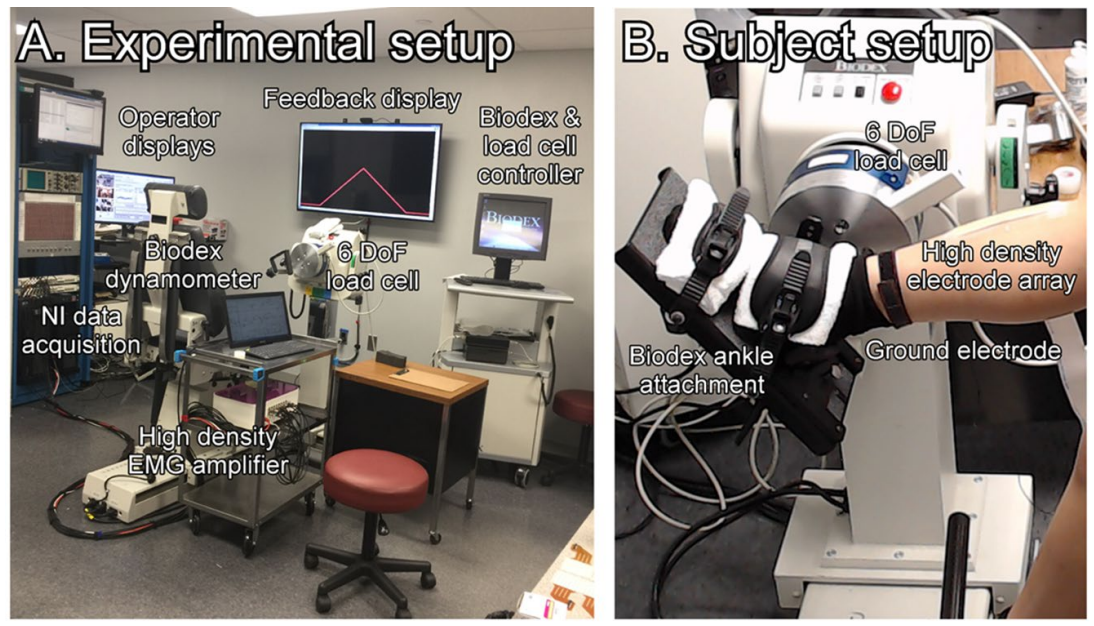

Figure 1. Experimental setup. A. Laboratory setup during data collection. Subject is seated in Biodex facing the feedback display. B. Sagittal view of the ankle during data collection for the TA. The axis of rotation of the ankle is lined up with the center of the load cell. 


\section{Experimental Set $U p-A r m$}

In a similar manner as described above, the skin over the dominant-side (i.e., right) first dorsal interosseous (FDI) was prepared and one 64-channel high-density electrode array (4mm interelectrode distance) was placed on the skin over the largest portion of the muscle belly and secured with medical tape. Participants were seated in a comfortable position in a chair facing an exam table. Chair height was adjusted such that their arms were resting in a neutral position on the exam table (forearm pronated, such that the palms were facing down). The tested hand was positioned against a rigid block on the exam table with fingers fully extended and thumb abducted $90^{\circ}$ away from the index finger. This position enabled the participant to exert abduction force with their index finger into the rigid block without moving the rest of their hand or fingers. Real-time feedback of FDI EMG was provided on a TV monitor, positioned directly in front of the participant, to assist task performance.

\section{Experimental Protocol}

At the beginning of each experimental session, participants performed three maximal volitional isometric contractions (MVIC) of the tested muscle, waiting at least two minutes between attempts, to establish a baseline of performance. For the purpose of EMG decomposition and subsequent motor unit analysis, participants were asked to produce a symmetrical time-varying isolated joint torque up to a fixed percentage (20\%) of their MVIC and at a consistent rate of torque development. Participants performed three sets of three isometric contractions for each muscle tested. To mitigate the effects of fatigue and windup, 10 seconds of rest between contractions and 2 minutes of rest between sets was imposed.

\section{Data Collection}

During isometric contractions, hdEMG data was recorded using the OTBioLab software (OT Bioelettronica; Turin, Italy). Torque was baseline corrected and filtered using a $20 \mathrm{~Hz}$ low pass filter, this signal was used for visual feedback of task performance during the TA contractions. A single channel of EMG was isolated, amplified, rectified, and smoothed using a $\sim 500$ ms root mean square filter, and then utilized for visual feedback of task performance during the FDI contractions. EMG was filtered at $20-900 \mathrm{~Hz}$ and collected at $2048 \mathrm{~Hz}$ using a 16-bit A/D converter (OT Bioelettronica; Turin, Italy), simultaneously with the torque data.

\section{Motor Unit Decomposition and Analysis}

hdEMG array recordings were decomposed offline into the discharge of individual motor units using a blind source separation algorithm ${ }^{18}$ (Figure 2). 


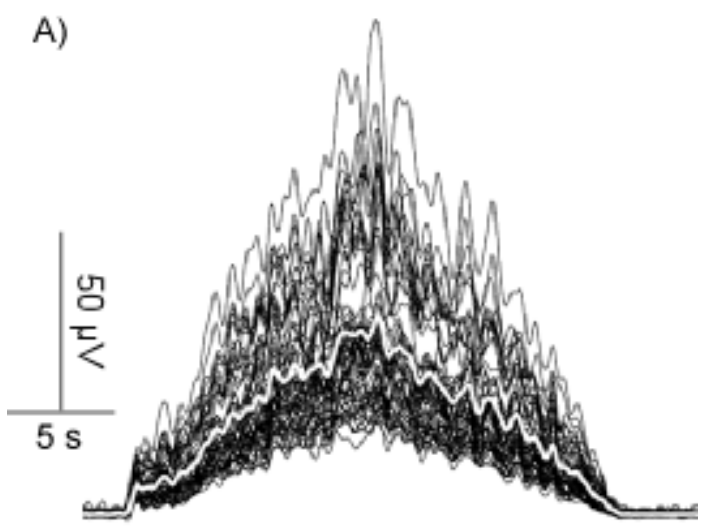

B)

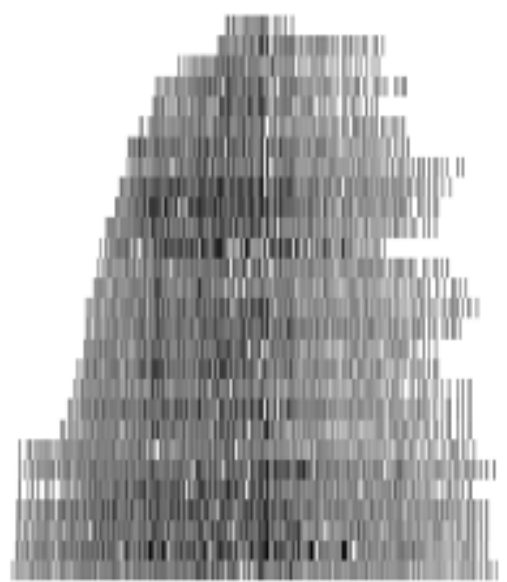

Figure 2. Conditioned EMG and decomposed MU spike trains. Representative data from the TA of one individual. A) Surface EMG is collected from 64 channel surface EMG during an isometric ramp contraction. B) Surface EMG recordings are decomposed into their corresponding motor unit action potentials; 28 motor units from the TA are shown here

This algorithm has been extensively validated and provides an accurate method for decomposing motor unit spike trains (e.g. Thompson et al. $^{20}$ ). For each participant, individual isometric contractions were isolated. Thereafter, each spike train was identified and convolved with a 2 -second hanning window (Figure 3). From these windowed spike times, the maximum discharge rate of each $\mathrm{MN}$ was calculated. 

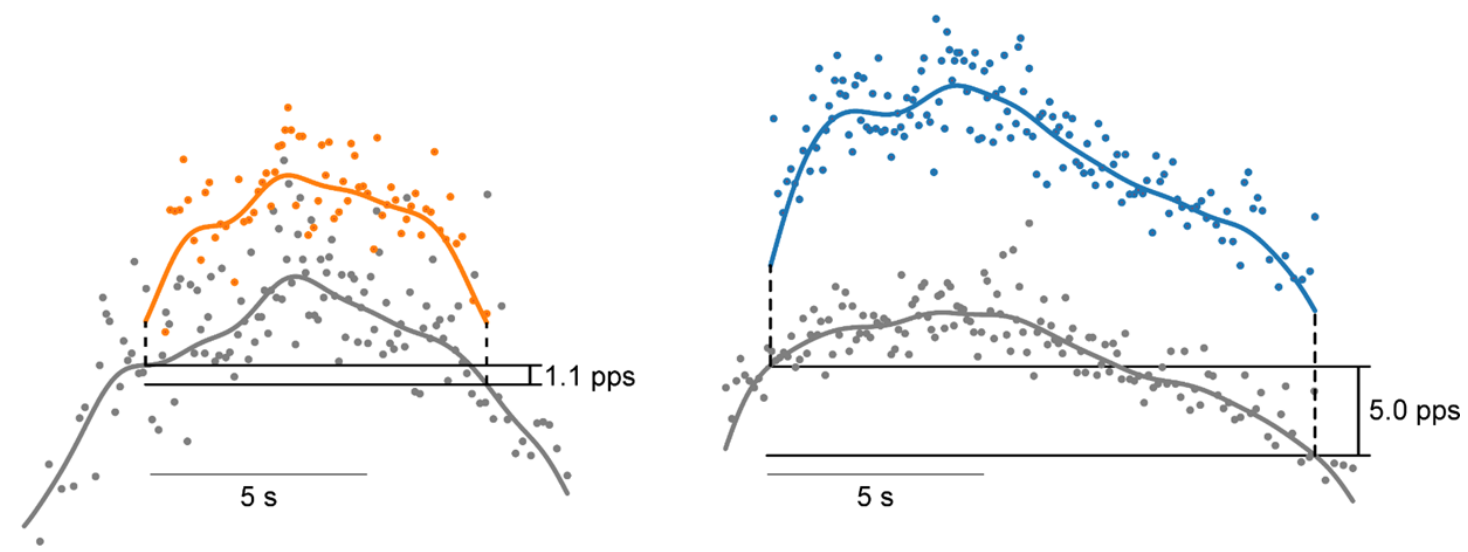

Figure 3. Paired motor unit analysis. Exemplar data from the paired motor unit analysis technique for the FDI (left) and TA (right); lower threshold motor units (gray) from the same contraction are paired with higher threshold units for analysis (FDI in orange; TA in blue). In this example, $\Delta \mathrm{F}$ is greater in the TA (right; $5.0 \mathrm{pps}$ ), as such, a larger hysteresis is observable and the higher threshold motor unit remains active for much longer when compared to its paired lower threshold motor unit. (Pulses per second; pps)

\section{Measurement of Intrinsic Motorneuron Excitability $(\Delta F)$}

PICs act to augment and prolong the activity of motor units. To estimate the magnitude of these effects, we measured the prolonged activity of motor units through the use of slow, symmetrically ramping (up to $20 \%$ MVIC and back down), isometric muscle contraction. If there were no appreciable nonlinear properties of these MNs, the time at which a motor unit was recruited and derecruited would be perfectly equal and symmetrical on the up- and down-slope of the triangular contraction. However, if there are increases in the excitability of spinal motoneurons (as such is the case with increased PICs), motor units would have an observable hysteresis, in which a motor unit would remain active long after it was expected to derecruit. Because it is not possible to directly assess the synaptic input to a motor pool from the nervous system in humans, a paired motor unit technique ${ }^{19}$ was utilized. Specifically, the recruitment and derecruitment time of a higher threshold unit was found and compared to the estimated synaptic current (i.e., the firing frequency of a lower threshold motor unit). $\Delta \mathrm{F}$ is calculated by finding the difference in discharge rate of the lower threshold unit between these two time points. In instances where $\Delta \mathrm{F}$ is greater, a larger hysteresis will become observable between the time at which a higher threshold unit is recruited and derecruited (see Figure 3). In an effort to limit the amount of erroneous comparisons between motor unit pairs, several criteria were employed. Firstly, the time between the first discharge of the lower and higher threshold units was less than $500 \mathrm{~ms}$. Secondly, to ensure a lack of saturation, an increase of at least $0.5 \mathrm{pps}$ in the lower threshold unit following the recruitment of the higher threshold unit was observed. Lastly, to ensure common synaptic drive underlying the rate modulation of both units, only pairs with a raterate $\mathrm{r} 2$ value of greater than 0.7 were considered suitable pairs. ${ }^{21}$ 


\section{Statistical Analysis}

Normality of the data was tested with the Shapiro-Wilk test and revealed non-normal distributions for $\Delta \mathrm{F}$ and maximal firing rate. Thus, Mann-Whitney $\mathrm{U}$ tests were used to compare the $\Delta \mathrm{F}$ and maximal firing rate within each subject between the TA and FDI. A Bonferroni correction was applied to account for multiple comparisons. All statistical analyses were performed using MATLAB 2017b (MathWorks, Natick, USA) and significance was accepted at the $\mathrm{p}$ value $\leq 0.05$. Cliff's Delta $(\Delta)$ was utilized to calculate effect size. ${ }^{22}$ Results are reported as median and [first quartile - third quartile].

\section{Results}

Each of the four subjects completed three sets of three contractions in both the TA and FDI for a total of 72 contractions. Figure 2 shows an example of a single contraction in which the hdEMG signals are overlaid above the decomposed discharge times. The TA contractions were performed at 18.4\% [15.8 - 22.1] maximal EMG amplitude while FDI contractions were performed at 23.8\% [21.1 - 27.7] maximal EMG amplitude. From these signals, a significant difference in the number of MUs yielded per contraction is observed between the TA (15.5 [12 - 21]) and FDI (5.5 [4 - 7]; $p=.006)$. All subjects (4/4) had a significantly higher maximum discharge rates in the FDI than in the TA (all $\mathrm{p}<0.01$ ). This ranged from a $15.1 \%$ to a $67.5 \%$ increase in discharge rate of the FDI as compared to the TA (Figure 4a). These differences represent a large effect size in discharge rate between FDI and TA (Cliff's $\Delta: 0.64-0.79)$.

To conduct the paired motor unit analysis, suitable motor unit pairs in each contraction were found (see methods). Figure 3 shows an example of the $\Delta \mathrm{F}$ analysis in pairs of motor units from the TA and FDI, note the greater hysteresis in the TA. This resulted in a median of 42 [11 - 101] pairs in the TA and 6 [4 - 16] pairs in the FDI per contraction. When pooling these values, all subjects (4/4) had a higher $\Delta \mathrm{F}$ in the TA (4.22 [2.89-5.61] pps) than FDI $(3.62$ [1.23 - 5.94] pps), with $3 / 4$ reaching statistical significance (Figure $4 \mathrm{~b})$. The effect size of the $\Delta \mathrm{F}$ between FDI and TA varied from small to moderate, among the four subjects (Cliffs $\Delta$ : $0.04-0.34$ ). 
A)

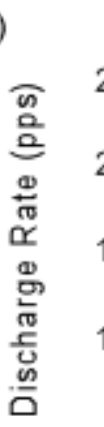

Subject 1

Subject 2

Subject 3

Subject 4
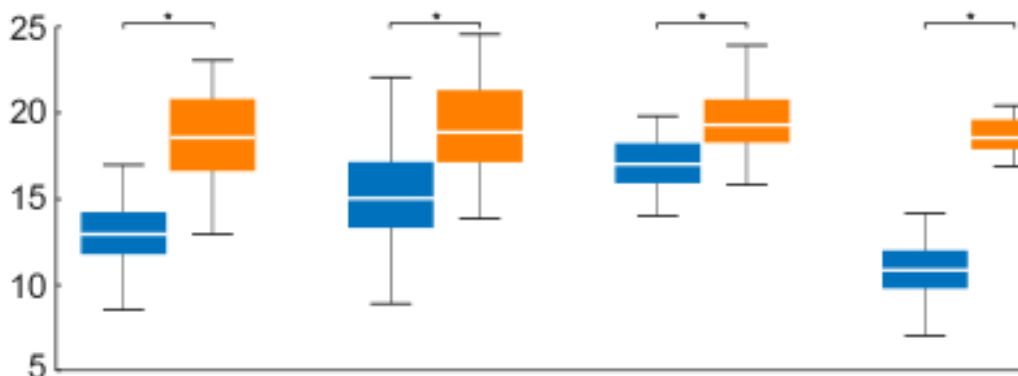

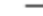

B)

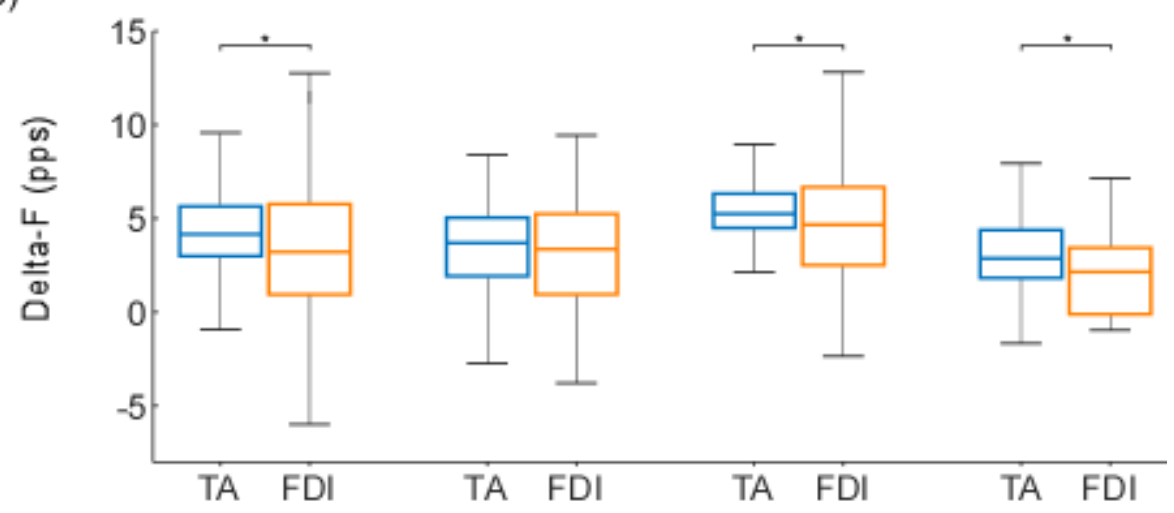

Figure 4. Individual data findings. Individual data (subjects 1-4) for maximal discharge rate (top row, filled boxes) and $\Delta \mathrm{F}$ (bottom row, empty boxes). Significant differences between TA (blue) and FDI (orange) are noted with $(*)$.

\section{Discussion}

The discharge patterns and intrinsic excitability of human MNs is different across functionally diverse motor pools. These findings indicate that the FDI preferentially utilizes higher discharge rates than the TA to achieve the same relative level of effort. Furthermore, using the delta-f approach, we observe the intrinsic excitability of human MNs is higher in the TA than in the FDI.

Two functionally diverse muscles appear to be controlled in fundamentally different ways by the CNS to achieve task dependent outcomes. This evidence supports the notion that motor pools are functionally tuned to their environmental demands. While others have observed state-dependent changes in the motor system through neuromodulation, ${ }^{24}$ none have yet assessed the baseline organization and relative sensitivity of these motor pools (TA and FDI) in humans. Because of the nonlinear ways in which PICs can augment motor unit behavior, it is possible that the nervous system may utilize these organizational differences to transform the motor command between motor pools, to facilitate complex motor tasks. For example, the TA, predominantly a postural control muscle, is utilized constantly for stability and locomotion. As such, its MNs may have a biophysical benefit to higher levels of excitability $(\Delta \mathrm{F})$, this would lower the effective activation threshold of the motor pool to 
assist in repetitive tasks. In contrast, the FDI is almost exclusively utilized for fine control in grasping. As such, its MNs may benefit from a reduced level of excitability when executing controlled, fine motor tasks, in which prolonged $\mathrm{MN}$ activation may increase the difficulty of controlling a precise movement.

In addition to differences in intrinsic $\mathrm{MN}$ excitability, during slow time varying isometric contractions the FDI preferentially utilizes higher discharge rates than the TA to achieve the same relative level of effort. The difference in maximal discharge rates could reflect further organizational differences between the TA and FDI. Others have reported similar findings in the characteristics and discharge rates of the $\mathrm{TA}^{24}$ and FDI. ${ }^{25}$ While the absolute size and maximal force output of the TA and FDI muscles may greatly vary, the use of a normalized task (20\% MVIC) acts to diminish these differences and alone are not likely responsible for the observed differences. However, relative differences in the amount of MNs and corresponding innervation ratio to muscle fibers, between these two motor pools could result in varying force-generating capabilities ${ }^{26}$ and, as a result, require divergent control strategies. The current data is consistent with previous work suggesting the FDI uses a rate-modulation (as opposed to recruitment) based strategy for increasing force. ${ }^{25}$ Taken together, the data suggest that distinct organizational differences exist between the TA and FDI motor pools.

It is important to note that consequent to the calculations involved in estimating intrinsic $\mathrm{MN}$ excitability with the paired motor unit method, the $\Delta \mathrm{F}$ value cannot exceed the peak discharge of the motor pool under observation. Thus, it is possible that the observed $\Delta \mathrm{F}$ findings in the TA may have been underestimated by their inherently lower discharge rates when compared to the FDI (with a higher discharge rate and lower $\Delta \mathrm{F}$ value). It is possible the differences between motor pools may be even larger without this limitation. This would be more of a concern if both the peak discharge rate and the $\Delta \mathrm{F}$ measure trended in the same direction, regardless this should be considered in its future use in comparing values across motor pools.

This method of hdEMG analysis generates an extraordinary amount of data per subject (in some cases hundreds of data points, per muscle, per contraction), in which important biophysical signals and individual variation may be lost when collapsing the data across subjects. Further, within the same subject, the TA and FDI may not have had the same number of representative data samples recorded in each motor pool. To address this unequal sampling, unpaired tests of statistical inference were chosen to assess changes within subjects. Additionally, due to a multitude of reasons including subcutaneous fat, anatomical differences, and signal noise, not all subjects yielded similar numbers of motor unit spike trains after decomposition. For example, subject 2 yielded a total of 145 motor unit comparisons for the paired motor unit analysis compared to subject 4 who yielded 847 . This disparity in motor unit comparisons may account for the non-significant increase in TA $\Delta \mathrm{F}$ in subject 2 (see Figure 4b). Future efforts should aim to improve motor unit spike train yield and to provide further rigor in statistical inference testing with this unique data collection method. 
Motor unit decomposition methods in hdEMG, such as the ones employed in this study, present multiple advantages in data collection over the lifespan in populations with neuromuscular pathology. Fine-wire EMG, in which a needle is inserted percutaneously into the muscle belly to temporarily place metal wires for direct recording and electrostimulation, is commonly used for diagnosis and prognosis across a range of neuromuscular pathologies, including amyotrophic lateral sclerosis (ALS) and peripheral nerve lesions (e.g., obstetric brachial plexus injury). However, the invasiveness of these procedures creates discomfort for vulnerable populations, deters patients from serial studies, requires trained clinician oversight, and presents analytical limitations. ${ }^{27}$ Instead, noninvasive hdEMG techniques can be employed, creating opportunities for remote testing in outpatient facilities, or even patients' homes, without the resources needed in a hospital or laboratory setting. The noninvasive nature of the hdEMG collection allows for longer recording periods with minimal discomfort and often yielding magnitudes more data. ${ }^{28}$ Fine-wire EMG, despite high levels of focal fidelity, cannot provide information about the entire motor pool during contractions. As a result, fine-wire EMG is unable to distinguish between individual MN activity and the behavior of the entire motor pool.

These findings extend the non-linear role of MNs in the animal model to humans (e.g., Hounsgaard \& Kiehn $^{29}$ ), suggest different levels of excitability across MN pools, ${ }^{13}$ and characterize non-linear MN behavior in healthy humans. High-density EMG and motor unit decomposition may be used in future research to map MN behavior (e.g., excitability and discharge rate) across motor pools in response to other conditions (e.g., modes, intensities, and intervals of activation). ${ }^{30}$ Understanding this behavior may have implications for the diagnosis, prognosis, and treatment of neuropathologies, including ALS, spinal cord injury, and stroke.

\section{Disclosures and Conflicts of Interest}

No conflicts of interest to report.

\section{Funding Sources}

This work was supported by NS098509 to Charles J. Heckman.

\section{Acknowledgments}

We wish to thank Francesco Negro for his expertise and assistance with the blind source separation algorithm. 


\section{References}

1. Heckman CJ, Enoka RM. Motor unit. Compr. Physiol. 2012 Oct; 2(4): 2629-2682.

2. Adrian ED, Bronk DW. The discharge of impulses in motor nerve fibres: Part II. The frequency of discharge in reflex and voluntary contractions. J Physiol. 1929 Mar 20;67(2):i3151.

3. Monster AW, Chan H. Isometric force production by motor units of extensor digitorum communis muscle in man. J Neurophysiol. 1977 Nov;40(6):1432-1443.

4. Henneman E. Relation between size of neurons and their susceptibility to discharge. Science. 1957 Dec 27; 26(3287):1345-1347.

5. Milner-Brown HS, Stein RB, Yemm R. Changes in firing rate of human motor units during linearly changing voluntary contractions. J Physiol. 1973;230(2):371-390.

6. Conway BA, Hultborn H, Kiehn O, Mintz I. Plateau potentials in alpha-motoneurones induced by intravenous injection of L-dopa and clonidine in the spinal cat. J Physiol. 1988 Nov;405:369-384.

7. Crone C, Hultborn H, Kiehn O, Mazieres L, Wigstrom H. Maintained changes in motoneuronal excitability by short-lasting synaptic inputs in the decerebrate cat. $J$ Physiol. 1988 Nov;405:321-343.

8. Hounsgaard J, Hultborn H, Jespersen B, Kiehn O. Bistability of alpha-motoneurones in the decerebrate cat and in the acute spinal cat after intravenous 5-hydroxytryptophan. J Physiol. 1988 Nov;405:345-367.

9. Perrier JF, Delgado-Lezama R. Synaptic release of serotonin induced by stimulation of the raphe nucleus promotes plateau potentials in spinal motoneurons of the adult turtle. $J$ Neuroci. 2005 Aug 31;25(35):7993-7999.

10. Heckman CJ, Lee RH, Brownstone RM. Hyperexcitable dendrites in motoneurons and their neuromodulatory control during motor behavior. Trends Neurosci. 2003 Dec;26(12):688-695.

11. Lee RH, Heckman CJ. Influence of voltage-sensitive dendritic conductances on bistable firing and effective synaptic current in cat spinal motoneurons in vivo. J Neurophysiol. 1996 Sep;76(3):2107-2110.

12. Bennett DJ, Hultborn H, Fedirchuk B, Gorassini M. Synaptic activation of plateaus in hindlimb motoneurons of decerebrate cats. J Neurophysiol. 1998 Oct;80(4):2023-2037.

13. Wilson JM, Thompson CK, Miller LC, Heckman CJ. Intrinsic excitability of human motoneurons in biceps brachii versus triceps brachii. J Neuroci. 2015 Jun 1;113(10):36923699.

14. Murray KC, Stephens MJ, Ballou EW, Heckman CJ, Bennett DJ. Motoneuron excitability and muscle spasms are regulated by 5-HT2B and 5-HT2C receptor activity. J Neurophysiol. 2011 Feb 1;105(2):731-348.

15. ElBasiouny SM, Schuster JE, Heckman CJ. Persistent inward currents in spinal motoneurons: Important for normal function but potentially harmful after spinal cord injury and in amyotrophic lateral sclerosis. Clin Neurophysiol. 2010 Oct;121(10):1668-1679.

16. McPherson J, Ellis,M, Heckman CJ, Dewald J. Evidence for increased activation of persistent inward currents in individuals with chronic hemiparetic stroke. J Neurophysiol. 2008 Dec;100(6):3236-3243.

17. Nardelli P, Vincent JA, Randall P, Cope TC, Rich MM. Reduced motor neuron excitability is an important contributor to weakness in a rate model of sepsis. Exp Neurol. 2016 Aug 1;282:1-8. 
18. Negro F, Muceli S, Castronovo AM, Holobar A, Farina D. Multi-channel intramuscular and surface EMG decomposition by convolutive blind source separation. J Neural Eng. 2016 Apr;13:026027.

19. Gorassini M, Yang JF, Siu M, Bennett DJ. Intrinsic activation of human motoneurons: Possible contribution to motor unit excitation. J Neurophysiol. 2002 Apr;87:1850-1858.

20. Thompson CK, Negro F, Johnson MD, Holmes MR, McPherson LM, Powers RK, Farina $\mathrm{D}$, Heckman CJ. Robust and accurate decoding of motoneuron behaviour and prediction of the resulting force output. J Physiol. 2018 Jul;596(14):2643-2659.

21. Gorassini MA, Knash ME, Harvey PJ, Bennett DJ, Yang JF. (2004). Role of motoneurons in the generation of muscle spasms after spinal cord injury. Brain. 2004 Oct;127(10):22472258.

22. Macbeth G, Razumiejczyk E, Ledesma R. Cliff's Delta Calculator: A non-parametric effect size program for two groups of observations. Univ. Psychol. [online]. 2011 May/Aug;10(2):545-555.

23. Wei K, Glaser JI, Deng L, Thompson CK, Stevenson IH, Wang Q, Hornby TG, Heckman CJ, Kording KP. Serotonin affects movement gain control in the spinal cord. J Neuroci. 2014 Sep 17;34(38):12690-12700.

24. Connelly DM, Rice CL, Roos MR, Vandervoort AA. Motor unit firing rates and contractile properties in tibialis anterior of young and old men. J Appl Physiol. 1999 Aug 1;87(2):843852.

25. Seki K, Narusawa M. Firing rate modulation of human motor units in different muscles during isometric contraction with various forces. Brain Res. 1996 May 6;719(1-2):1-7.

26. Lawrence JH, De Luca CJ. Myoelectric signal versus force relationship in different human muscles. J Appl Physiol Respir Environ Exerc Physiol. 1983 Jun;54(6):1653-1659.

27. Bashford J, Mills K, Shaw C. The evolving role of surface electromyography in amyotrophic lateral sclerosis: A systematic review. Clin Neurophysiol. 2020 Apr;131(4):942-950.

28. Farina D, Holobar A, Merletti R, Enoka RM. Decoding the neural drive to muscles from the surface electromyogram. Clin Neurophysiol. 2010 May 4;121(10):1616-1623.

29. Hounsgaard J, Kiehn O. Serotonin-induced bistability of turtle motoneurones caused by a nifedipine-sensitive calcium plateau potential. J Physiol. 1989 Jul;414:265-282.

30. Enoka RM. Physiological validation of the decomposition of surface EMG signals. $J$ Electromyogr Kinesiol. 2019 Jun;46:70-83.

\section{Statement of Contributions}

Christopher K. Thompson designed the study. Christopher A. Taylor and Tyler Kmiec performed the experiments and processed the data. Christopher A. Taylor and Christopher K. Thompson analyzed the data. Christopher A. Taylor prepared the initial draft of the manuscript. Christopher A. Taylor, Tyler Kmiec and Christopher K. Thompson reviewed, contributed to, and approved the final manuscript. 